\title{
RITMO SAMBA COMO ESTIMULAÇÃO AUDITIVA RÍTMICA E DESEMPENHO DA MARCHA NA DOENÇA DE PARKINSON
}

\section{Samuel Geraldi Fragnani}

Discente do curso de graduação em Fisioterapia da Universidade Federal de Santa Catarina (UFSC), Brasil.

\section{Poliana Penasso Bezerra}

Docente do Curso de graduação em Fisioterapia, Departamento de Ciências da Saúde, Centro de Ciências, Tecnologias e Saúde - Universidade Federal de Santa Catarina (UFSC), Araranguá, Brasil.
Autor correspondente

Poliana Penasso Bezerra

E-mail: poliana.bezerra@ufsc.br
RESUMO: A estimulação auditiva rítmica tem sido utilizada para amenizar as disfunções motoras na doença de Parkinson, no entanto não são evidentes as alterações que a música popular brasileira causa nos parâmetros espaço-temporais e desempenho funcional da marcha. $O$ objetivo do estudo foi analisar o gênero musical samba durante a marcha de pacientes com doença de Parkinson em situações de dupla tarefa. Estudo de caráter transversal, descritivo e exploratório; cinco indivíduos atenderam aos critérios propostos e realizaram o Timed Up and Go Test nas situações sem música e música popular brasileira (samba) sensibilizadas com tarefas cognitivas e motoras para análise da marcha. Análise estatística descritiva e inferencial com teste de Fridman e post-boc de Wilcoxon pareado (nível de significância de $5 \%$ ). Evidenciou-se que o samba associado a uma tarefa motora reduziu a velocidade $(p=0,01)$ e o comprimento de passos $(p=0,04)$ quando comparados à condição de teste sem música. Ouvir música proporcionou maiores demandas atencionais, resultando na adoção de estratégias para diminuir as exigências de equilíbrio postural como a redução do comprimento do passo e velocidade e desta forma diminuir o risco de quedas em situações de duplas tarefas enquanto escuta música.

PALAVRAS-CHAVE: Música; Marcha; Doença de Parkinson.

\section{SAMBA RHYTHM FOR RHYTHMIC HEARING STIMULATION AND GAIT PERFORMANCE IN PARKINSON'S DISEASE}

\begin{abstract}
Rhythmic hearing stimuli have been employed to attenuate motor dysfunctions in Parkinson's disease. However, changes that may be caused in space-time parameters and functional performance of gait brought about by Brazilian popular music are not always evident. Current analysis deals with the musical genre samba during gait of patients suffering from Parkinson's disease in a twotask situation. Current transversal, descriptive and exploratory study comprised five agents who fulfilled criteria and undertook the Timed Up and Go Test in situations without music and with Brazilian popular music (samba) through cognitive and motor tasks for gait analysis. Descriptive and inferential statistical analysis with Friedman's test and with paired Wilcoxon post-hoc test (at 5\% significance) evidenced that samba associated with a motor task reduced the speed $(p=0.01)$ and gait length $(p=0.04)$ when compared to test conditions without any music. Listening to music demanded more attention and resulted in the use of strategies to decrease demands of posture balance, such as reduction in gait length and speed. Decrease in falling risks in a twotask situation while listening to music may occur.
\end{abstract}




\section{INTRODUÇÃO}

Atualmente a marcha é considerada como um movimento sincronizado, automático e cíclico que permite o deslocamento do corpo no espaço ${ }^{1,2}$. Embora este seja um movimento automatizado, o controle da marcha é um processo complexo que consiste na decodificação dos estímulos aferentes pelo córtex sensorial, na interpretação das informações no córtex associativo e por fim na resposta motora cortical com a participação dos núcleos da base para resposta eferente muscular ${ }^{3,4}$

Percebe-se com isso que os núcleos da base desempenham importante papel para a realização do movimento. No entanto algumas doenças podem causar alterações neste mecanismo, entre elas a doença de Parkinson (DP). Esta disfunção consiste em um dos mais crescentes distúrbios neurológicos do mundo e afeta principalmente os idosos 5 . Pela redução do neurotransmissor dopamina no corpo estriado, os indivíduos com DP podem ter alteração nos movimentos voluntários ou automáticos ${ }^{6}$.

Desta forma, a DP cria nas estruturas cerebrais um grande desafio para coordenar os movimentos da marcha. A doença também é caracterizada por ser progressiva, neurodegenerativa e com manifestações como rigidez muscular, tremor e déficit de equilíbrio postural. Além destas disfunções, a DP apresenta prejuízo nos domínios cognitivos sendo muitas vezes necessário realizar planejamentos e associações para o melhor desempenho motor destes processos depreciados ${ }^{5.7}$.

A estimulação auditiva rítmica, como ouvir música durante a marcha, tem sido a proposta como alternativa para amenizar as disfunções motoras depreciadas por doenças neurológicas ${ }^{3,8}$. Recentes estudos referem que esta é uma técnica eficaz para a reabilitação da marcha em pacientes com DP9,10. A fundamentação de seu uso está estabelecida nos princípios de aprendizagem motora e nas teorias neurofisiológicas dos processos cognitivos ${ }^{11}$.

Uma estratégia para utilização destes fundamentos consiste na intervenção fisioterapêutica realizada dentro da faixa de ritmo perceptível dos seres humanos ( 40 a 300 batidas por minuto) $)^{3}$. Nesta proposta de intervenção emprega-se um ritmo confortável como estimulação auditiva rítmica para adequar os parâmetros espaço-temporais da marcha. Sendo assim, esta conexão entre ritmo e parâmetros da marcha pode justificar o uso clínico da estimulação auditiva rítmica nos processos de reabilitação da marcha em pacientes com doenças neurológicas ${ }^{12}$.

No entanto, a marcha associada à realização de tarefas simultâneas como ouvir música, requer a divisão de atenção e de estratégias de função executiva não sendo claro quais as estratégias adotadas por estes indivíduos. Da mesma forma, pouco se questiona em relação às alterações que os parâmetros espaço-temporais sofrem quando submetidos à estimulação auditiva rítmica proporcionadas pela música. Além desses itens, também não é evidente a relação desempenho funcional e interpretação linguística que a música popular brasileira possa causar simultaneamente às atividades cotidianas. O estudo objetiva analisar o efeito da estimulação auditiva rítmica do gênero musical samba na marcha de pacientes com DP durante a execução da dupla tarefa, visto que as atividades de vida diária associadas à música é hábito comum nos dias atuais. Pressupomos que a estimulação auditiva rítmica possa modificar os padrões de planejamento motor durante a marcha de pacientes com DP a fim de promover adequações nos parâmetros espaço-temporais da marcha e execução de dupla tarefa.

\section{MÉTODO}

Trata-se de um estudo de caráter transversal, descritivo e exploratório, composto por grupo de indivíduos com diagnóstico clínico de DP provenientes da Associação de Parkinson, Araranguá-SC, Brasil. A pesquisa foi aprovada pelo Comitê de Ética em Pesquisa com Seres Humanos da Universidade Federal de Santa Catarina, conforme parecer número 1.147.333).

Foram incluídos indivíduos com diagnóstico clínico de DP, que estavam em uso regular da medicação, classificados entre os estágios 1 e 4 na escala de Hoehn e $\mathrm{Yahr}^{13} \mathrm{e}$ que concordassem em assinar o termo de consentimento livre e esclarecido de acordo com os critérios estabelecidos. Foram excluídos aqueles que apresentassem outras patologias neurológicas associadas, estimulador cerebral profundo, distúrbios auditivos, aqueles que estivessem em período de adaptação à medicação, que fossem incapazes de deambular de forma independente e que não compareceram às intervenções propostas. Dos 11 indivíduos potenciais para o estudo, cinco foram incluídos. Na Figura 1 é apresentado o fluxograma de inclusões e exclusões amostrais. 




Figura 1. Fluxograma de inclusão e exclusão da amostra

O perfil demográfico dos indivíduos foi caracterizado conforme sexo, idade, estadiamento da doença analisado pela escala de Hoehn e Yahr ${ }^{13}$, progressão da doença pela escala Unificada de Avaliação da Doença de Parkinson (UPDRS) ${ }^{14}$, cognição pelo MiniExame do Estado Mental (MEEM) ${ }^{15}$ e Avaliação Cognitiva Montreal (MoCA $)^{16}$, equilíbrio pela escala de Equilíbrio Berg $(\mathrm{EEB})^{17} \mathrm{e}$ funcionalidade pela escala de Atividade de Parkinson (PAS) ${ }^{18}$.

A escala de Hoehn e Yahr avalia e classifica a doença em cinco estágios. Sua avaliação abrange medidas globais de sinais e sintomas que permitem qualificar o indivíduo quanto ao nível de incapacidade, instabilidade postural, rigidez, tremor e bradicinesia ${ }^{13}$. A UPDRS avalia a progressão da doença de acordo com suas características clínicas, composta por 42 itens, divididos em quatro domínios (atividade mental, comportamento e humor, atividades de vida diária, exploração motora e complicações da terapia medicamentosa). A pontuação em cada item varia de 0 a 4 , sendo que quanto maior a pontuação, maior o comprometimento da doença ${ }^{14}$.

O MEEM avalia as funções cognitivas, composto por questões agrupadas em sete categorias, cada uma delas com o objetivo de avaliar funções cognitivas específicas, como orientação para tempo e espaço, registro e lembrança de palavras, linguagem, atenção, cálculo e capacidade construtiva visual. O escore pode variar de 0 a 30 pontos, e o ponto de corte 24 tem de boa a excelente sensibilidade e especificidade para 0 diagnóstico de demência ${ }^{15}$. A MoCA acessa os domínios cognitivos da atenção e concentração, funções executivas, memória, linguagem, habilidades viso-construtiva, conceituação, cálculo e orientação sendo a pontuação máxima de 30 pontos $^{16}$.
O equilíbrio e controle postural foram avaliados com a EEB que compreende uma escala de 14 tarefas relacionadas ao dia a dia, que envolvem o equilíbrio estático e dinâmico, tais como alcançar, girar, transferirse, permanecer em pé e levantar-se. Os itens avaliados incluem a habilidade do indivíduo em manter posições de crescente dificuldade, com a diminuição da base de suporte para sentar, até postura confortável, ficar em pé com os pés juntos, e por final, postura em tandem e postura unipodal. Outros itens avaliam o quão bem o indivíduo está apto a mudar de posição, de sentado para em pé, ao baldear-se de uma cadeira para outra, ao pegar um objeto do piso e ao sentar-se. A realização das tarefas é avaliada por meio da observação e a pontuação varia de 0 a 4 em cada tarefa, totalizando um máximo de 56 pontos, e a pontuação é baseada no tempo em que a posição pode ser mantida, a distância que o braço é capaz de alcançar para a frente, ou o tempo para completar uma tarefa. Cada ponto a menos está associado a um aumento no risco de quedas ${ }^{17}$.

A PAS é usada para descrever e quantificar as alterações de mobilidades dos pacientes com DP e baseiase em dez tarefas que esses indivíduos costumam ter dificuldade, divididas em quatro domínios: transferências na cadeira, marcha, mobilidade no leito e mobilidade no leito com cobertas. $\mathrm{O}$ escore varia de 0 a 40 , sendo que quanto menor a pontuação, maior a dificuldade no desempenho de tarefas de mobilidade, e, portanto, pior o nível de mobilidade do indivíduo ${ }^{18}$.

O desempenho motor na marcha dos participantes foi analisado por meio do teste de levantar e andar cronometrado (timed up and go test - TUG). Este teste de equilíbrio e mobilidade envolve manobras funcionais como levantar-se, caminhar, dar uma volta e sentar-se. O paciente foi cronometrado enquanto se levanta de uma cadeira (de aproximadamente $46 \mathrm{~cm}$ ), caminha em uma linha reta de 3 metros de distância (em ritmo confortável e seguro), vira, caminha de volta e senta-se sobre a cadeira novamente. O paciente realizou o percurso para se familiarizar com o teste antes de iniciálo. Orientou-se o uso de calçado habitual. Sua realização em até 10 segundos é considerada normal e indica baixo risco de quedas ${ }^{19}$.

Realizado em três condições: (1) TUG simples sem associação de nenhuma tarefa; (2) TUG sensibilizado com uma tarefa cognitiva (TUGcog). Este teste consistiu em andar e evocar maior quantidade de animais possíveis 
sem repetir ou utilizar do plural; (3) TUG sensibilizado com uma tarefa motora (TUGmot). Este teste consistiu em transferir maior quantidade de objetos de diferentes formas e tamanhos de uma bolsa plástica de mercado para outra bolsa plástica. Cada bolsa permanece em um dos membros superiores sendo livre a estratégia do paciente para realizar a tarefa proposta ${ }^{20}$.

As três condições de teste foram novamente realizadas sensibilizadas com o gênero musical samba por meio da música "É Hoje o dia do Grêmio Recreativo Escola de Samba União da Ilha do Governador". As três condições de teste foram realizadas com intervalo de sete dias entre elas e a ordem de execução dos testes definida aleatoriamente por meio de sorteio. Os testes foram realizados cinco vezes, sendo a primeira e a última excluída a fim de anular o efeito aprendizado.

Os parâmetros espaço-temporais da marcha foram avaliados durante os testes. A velocidade foi calculada pela variação do espaço e tempo cronometrado, comprimento de passo pela quantidade de passo e distância percorrida e a cadência pela quantidade de passos dados por tempo ${ }^{21}$.

Os testes foram realizados em ambiente bem iluminado, com pista livre de obstáculos, sons externos diminuídos e sem interferências de terceiros. Os testes iniciaram somente após o total entendimento da tarefa a ser cumprida pelo paciente. O gênero musical foi transmitido por meio de fones de ouvido intra-auriculares, modelo SJYN1181B e a quantidade de passos registrada por uma câmera fotográfica digital Nikon D3100®.

A análise estatística descritiva (média e desviopadrão) e inferencial foi realizada por meio do programa SPSS $^{21}$. Os dados foram avaliados para distribuição de normalidade pelo teste de Shapiro-Wilk. Optou-se pela aplicação do teste não paramétrico de Friedman em função de os mesmos indivíduos ser avaliados em três situações distintas (amostras emparelhadas). Para o post boc utilizou-se o teste Wilcoxon pareado. Foi adotado o valor $p<0,05$ para que os dados fossem considerados estatisticamente significativos.

\section{RESULTADO}

As características demográficas dos participantes estão demonstradas na Tabela 1 . A média de idade dos indivíduos foi de 58,8 $\pm 8,53$ anos, sendo quatro do sexo feminino e um masculino. Destes, um foi classificado no estágio 1 da escala de Hoehn e Yahr, três classificados no estágio 3 e um classificado no estágio 4, sendo o escore médio $2,8 \pm 1,10$ pontos.

A média verificada na escala UPDRS foi de $34,6 \pm 12,74$ pontos, verificando-se que os indivíduos do presente estudo apresentaram baixo comprometimento clínico da doença. Na aplicação da EEB, obtiveramse $53,4 \pm 2,79$ pontos, ratificando que os indivíduos apresentaram baixos problemas de equilíbrio e risco de queda. Na escala PAS foram obtidos 32,6 $\pm 9,36$ pontos, indicando independência funcional.

Em relação à escala MEEM, todos os participantes apresentaram escore superior a 24 pontos, sendo a média do teste de 26,8 $\pm 2,05$ e na MoCA, o escore médio foi de 23,4 \pm 4,62 pontos, sendo 30 pontos a máxima pontuação que pode ser obtida. Quando analisada a escolaridade, percebe-se que $80 \%$ possuem primeiro grau completo e $20 \%$ grau superior completo, verificando-se que essa amostra não apresentou declínio cognitivo.

O estímulo auditivo rítmico por meio do gênero musical samba promoveu alterações significativas nos parâmetros espaço-temporais reduzindo a velocidade $(p=0,01)$ e o comprimento de passos $(p=0,04) \mathrm{em}$ relação à condição sem música durante a execução de tarefa motora associada. Durante a execução de tarefa cognitiva associada não foi verificada interferência significativa (Tabela 2).

\section{DISCUSSÃO}

O presente estudo avaliou o efeito do estímulo auditivo rítmico no desempenho motor da marcha simultânea à atividade cognitiva e motora.

Os resultados demonstram que a dupla tarefa motora em todas as condições de teste diminuiu significativamente o parâmetro velocidade apenas quando realizado sob influência do ritmo samba $(p=0,01)$. Semelhante à velocidade, o comprimento de passos também foi reduzido quando os pacientes realizaram o teste de dupla tarefa motora associado a este gênero musical $(p=0,04)$.

Este desempenho pode ser explicado pela própria condição patológica da doença que promove rigidez muscular caracterizada pela contração entre os músculos agonista e antagonista. Além disso, as alterações dos núcleos da base alteram o controle da velocidade do movimento voluntário que está diminuído nos indivíduos 
com DP quando comparados a indivíduos saudáveis. Desta forma, as disfunções da marcha na DP podem ser reflexo da dificuldade em regular internamente o sistema de controle motor como o comprimento de passo e a velocidade da marcha ${ }^{3}$.

Segundo Souza ${ }^{22}$, esta dificuldade apresentada por indivíduos com DP que ocorre principalmente durante a dupla tarefa não é devida apenas à perda da regulação interna como a automaticidade dos movimentos, mas também às deficiências executivas relacionadas à atenção e à memória operacional. Estas disfunções fazem com que pessoas com a DP tenham aumento da dependência em recursos cognitivos para controlar seus movimentos. Desta forma, a associação de uma ou mais tarefas durante a marcha pode demandar de muitos recursos cognitivos levando a alterações no caminhar ${ }^{23}$.

Segundo Peterson e colaboradores ${ }^{24}$, as típicas mudanças no padrão da marcha também consistem na privação da capacidade do indivíduo de produzir um ritmo interno. Wu e Hallett ${ }^{23}$ mencionam em seu estudo de imagem por ressonância magnética que pessoas com DP, em situações de DT, têm maiores ativações nas áreas corticais em comparação com pessoas sem DP. No entanto, resultados de meta-análise, realizada com 66 estudos, demonstram que a alteração da marcha como diminuição da velocidade na DT possui forte ligação com a idade e ocorre em pacientes com ou sem a DP ${ }^{25}$. Por esta meta-análise entende-se que a mudança nos parâmetros da marcha consiste em, adaptação de caráter fisiológica em indivíduos submetidos a situações de DT ${ }^{25}$.

Sabe-se que a velocidade da marcha é diretamente proporcional ao comprimento do passo, sendo o maior comprimento de passo referente a uma maior velocidade. Também se entende que uma marcha com maior velocidade requer maior controle do equilíbrio pela redução no tempo de duplo apoio, além das rápidas acelerações do centro de massa do indivíduo ${ }^{26}$. Nas análises do presente estudo, o comprimento de passo e a velocidade tiveram significativa diminuição quando associado a tarefa motora durante o gênero musical samba.

Sugere-se que a diminuição do comprimento do passo e a lentificação da velocidade da marcha representam uma estratégia adotada pelos indivíduos com DP para diminuir as exigências de equilíbrio postural. Desta forma, por apresentar na situação imposta uma marcha mais lenta, com menor aceleração do centro de gravidade e maior tempo de duplo apoio, os pacientes tendem a diminuir o risco de quedas, tornando a marcha mais segura. A atenção dividida pela música pode ser usada como uma estratégia para melhorar o equilíbrio em condições de dupla tarefa ${ }^{27}$.

As alterações dos parâmetros da marcha durante a DT, observadas no presente estudo, também podem ser explicadas pela competição dos recursos atencionais entre processamento motor para marcha, realização da tarefa motora simultânea, o estímulo auditivo ritmico e interpretação linguística da música. A competição faz com que o processamento de uma tarefa seja temporariamente suspenso resultando em diminuição do desempenho da tarefa não priorizada. No entanto, não está claro na literatura qual tarefa, cognitiva ou motora, tem maior impacto na marcha em pessoas com DP ${ }^{28,29}$. Corroborando com nossos achados, estudos ${ }^{9,30}$ evidenciaram maiores impactos de tarefas motoras associadas na velocidade e comprimento de passo.

No presente estudo não foi encontrado alteração significativa no parâmetro cadência, no entanto, achados de Aria e Cudeiro ${ }^{31}$ demonstram que a estimulação auditiva rítmica aplicada em indivíduos com DP gerou efeito benéfico minimizando o efeito de congelamento e promovendo aumento da velocidade e cadência em todos os participantes do estudo. A estimulação auditiva rítmica tem sido estudada uma vez que o tempo de reação para sinais auditivo é 20-50 ms mais curtos do que para pistas visuais ou tácteis ${ }^{32}$.

Nos achados de Brown e colaboradores', a música imposta também modificou os parâmetros espaçotemporais da marcha dos indivíduos com DP. Os autores não orientaram os participantes a priorizar a música, no entanto é provável que os pacientes tenham ativamente focado a atenção na música. Desta forma, escutar música pode ter agido como uma tarefa adicional, criando uma situação de dupla tarefa que é especialmente difícil para pacientes com DP.

Em estudo com estimulação auditiva ritmica semelhante a este, Benoit e colaboradore ${ }^{33}$ realizaram treino de caminhada utilizando uma música folclórica alemã, sua amostra foi composta por 15 pacientes com DP não dementes, classificados com Hoehn e Yahr 2. No entanto, sua intervençãofoi decaráter longitudinal durante um mês, sendo realizadas três sessões de treinamento de 30 minutos por semana. Nestas sessões, os pacientes não 
receberam instruções explícitas para sincronizar seus passos ao rítmo da música, mesmo assim os participantes caminharam no ritmo das batidas mais salientes da música folclórica. Seus resultados demonstram que os pacientes com DP tiveram melhora significativa no comprimento de passo, velocidade e distância de marcha durante as sessões de treinamento. Além deste benefício, a melhora da marcha foi sustentada por um mês pós-treinamento, indicando um efeito terapêutico duradouro.

Este estudo apresentou limitações. Pelos critérios de inclusão e exclusão propostos, a amostra foi composta de cinco indivíduos com DP, podendo ser considerada pequena e pouco representativa, porém este estudo apresenta evidências científicas que fomentam a discussão sobre os efeitos imediatos positivos e negativos do estímulo auditivo rítmico no desempenho da marcha nesses pacientes. Ressaltamos, também, que a interpretação linguística da música escolhida pode ter sido diferente para cada paciente e isso não foi controlado no presente estudo.

No presente estudo, os testes avaliaram o impacto imediato que a música promoveu no desempenho da marcha, não possibilitando tempo suficiente para a aprendizagem motora. Desta forma, sugere-se que futuros estudos realizem a intervenção de caráter longitudinal com interpretação linguística do estímulo auditivo rítmico controlado.

A utilização do gênero musical samba na prática clínica poderia ser útil no treinamento da alocação de recursos atencionais e das estratégias adotadas por estes indivíduos no desempenho da marcha. Essas estratégias poderiam reduzir o risco de quedas durante o caminhar em situações cotidianas de duplas tarefas enquanto escuta música.

\section{CONCLUSÃO}

Em condições de dupla tarefa motora, o gênero musical samba promoveu alterações nos parâmetros espaço-temporais da marcha em relação à condição sem nenhuma estimulação auditiva. Dessa forma, ouvir música em situações de duplas tarefas pode ser considerado uma atividade que exige atenção adicional para os pacientes com DP, o qual desenvolve redução da velocidade e do comprimento dos passos para conseguir realizar a marcha com maior segurança e menor risco de queda e a tarefa simultânea.

\section{REFERÊNCIAS}

1. Zivotofsky AZ, Hausdorff JM. The sensory feedback mechanisms enabling couples to walk synchronously: an initial investigation. J Neuroeng Rehabil. 2007 Aug; $4: 28$.

2. Scaglioni-Solano P, Aragón-Vargas LF. Age-related differences when walking downhill on different sloped terrains. Gait Posture. 2015 Jan;41(1):153-8. doi:10.1016/j.gaitpost.2014.09.022.

3. Rochester L, Hetherington V, Jones D, Nieuwboer A, Willems AM, Kwakkel G, Van Wegen E. The effect of external rhythmic cues (auditory and visual) on walking during a functional task in homes of people with Parkinson's disease. Arch Phys Med Rehabil. 2005 May;86(5):999-1006.

4. Anson E, Agada P, Kiemel T, Ivanenko Y, Lacquaniti F, Jeka J. Visual control of trunk translation and orientation during locomotion. Exp Brain Res. 2014 Jun;232(6):1941-51.

5. O'Callaghan C, Lewis SJG. Cognition in Parkinson's Disease. Int Rev Neurobiol. 2017;133:557-83. doi: 10.1016/bs.irn.2017.05.002.

6. Rodríguez-Violante M, Camacho-Ordoñez A, Cervantes-Arriaga A, González-Latapí P, VelázquezOsuna S. Factors associated with the quality of life of subjects with Parkinson's disease and burden on their caregivers. Neurologia. 2015 Jun;30(5):257-63. doi: 10.1016/j.nrl.2014.01.008.

7. Bueno MEB, Andrello ACR, Terra MB, Almeida IA, Lemes LB, Batistelli CB, et al. Efetividade da fisioterapia com treinamento de dupla tarefa no sistema motor e cognitivo em indivíduos com doença de Parkinson. Revista Saúde e Pesquisa 2014;7(2):241-9.

8. Almeida BM, Silva FEB, Santos A, Madureira JR, Machado TPG. Efeito das atividades rítmicas nas habilidades motoras de paciente atáxico: um estudo de caso. R. Bras. Ci. e Mov. 2013; 21(3):71-9. doi: http:// dx.doi.org/10.18511/0103-1716/rbcm.v21n3p71-79

9. Brown LA, de Bruin N, Doan JB, Suchowersky O, Hu B. Novel challenges to gait in Parkinson's disease: the effect of concurrent music in single- and dual-task contexts. Arch Phys Med Rehabil. 2009 Sep;90(9):157883. doi:10.1016/j.apmr.2009.03.009. 
10. Thaut CP. Handbook of neurologic music therapy, 1ed. New York: Oxford University Press; 2014.

11.Carmeli E. Physical Therapy for Neurological Conditions in Geriatric Populations. Front Public Health. 2017;5:333. doi: 10.3389/fpubh.2017.00333.

12. François C, Grau-Sánchez J, Duarte E, RodriguezFornells A. Musical training as an alternative and effective method for neuro-education and neurorehabilitation. Front Psychol. 2015;6:475. doi: 10.3389/fpsyg.2015.00475.

13. Hoehn MM, Yahr MD. Parkinsonism: onset, progression and mortality. Neurology. 1967;17(5):427-42.

14.Fahn S, Elton RL. Unified Parkinson's disease rating scale. In: Fahn S, Goldstein M, Marsden D, Calne DB, editors. Recent developments in Parkinson's disease. New Jersey: MacMillan; 1987. p. 153-63.

15. Folstein MF, Folstein SE, McHugh PR."Mini-mental state": a practical method for grading the cognitive state of patients for the clinician. J Psychiatr Res. 1975;12(3):189-98.

16. Freitas S, Simões MR, Alves L, Santana I. Montreal Cognitive Assessment (MoCA): normative study for the Portuguese population. J Clin Exp Neuropsychol. 2011;33(9):989-96. doi: 10.1080/13803395.2011.589374.

17. Miyamoto ST, Lombardi Junior I, Berg KO, Ramos LR, Natour J. Brazilian version of the Berg balance scale. Braz J Med Biol Res. 2004;37(9):1411-21.

18. KeusSH,NieuwboerA,BloemBR,BormGF,MunnekeM. Clinimetric analyses of the Modified Parkinson Activity Scale. Parkinsonism Relat Disord. 2009;15(4):263-9. doi: 10.1016/j.parkreldis.2008.06.003.

19. Mathias S, Nayak US, Isaacs B. Balance in elderly patients: the "get-up and go" test. Arch Phys Med Rehabil. 1986;67(6):387-9.

20. Bloem BR, Valkenburg VV, Slabbekoorn M, Willemsen MD. The Multiple Tasks Test: development and normal strategies. Gait Posture. 2001;14(3):191-202.

21. O'sullivan SB, Schmitz TJ. Fisioterapia: avaliação e tratamento. São Paulo: Manole; 2010.

22. Souza CB. Efeitos de um treinamento em condição de dupla-tarefa sobre o desempenho motor e habilidade de dividir a atenção em pacientes com Doença de
Parkinson. 2008. Tese de mestrado. Universidade de São Paulo, São Paulo, 2008.

23. Wu T, Hallett M. Neural correlates of dual task performance in patients with Parkinson's disease. J Neurol Neurosurg Psychiatry. 2008;79(7):760-6.

24.Peterson DS, Plotnik M, Hausdorff JM, Earhart GM. Evidence for a relationship between bilateral coordination during complex gait tasks and freezing of gait in Parkinson's disease. Parkinsonism Relat Disord. 2012;18(9):1022-6. doi:10.1016/j. parkreldis.2012.05.019.

25. Al-Yahya E, Dawes H, Smith L, Dennis A, Howells $\mathrm{K}$, Cockburn J. Cognitive motor interference while walking: a systematic review and meta-analysis. Neurosci Biobehav Rev. 2011;35(3):715-28. doi: 10.1016/j.neubiorev.2010.08.008.

26. Neumann DA. Cinesiologia do Aparelho Musculoesquelético - Fundamentos Para a Reabilitação Física. 2. ed.Amsterdã: Elsevier; 2011.

27. Fok P, Farrell M, McMeeken J. The effect of dividing attention between walking and auxiliary tasks in people with Parkinson's disease. Hum MovSci. 2012;31(1):23646. doi: 10.1016/j.humov.2011.05.002.

28. Kelly VE, Eusterbrock AJ, Shumway-Cook A. A review of dual-task walking deficits in people with Parkinson's disease: motor and cognitive contributions, mechanisms, and clinical implications. Parkinsons Dis. 2012;2012:918719. doi: 10.1155/2012/918719.

29. Yogev-Seligmann G, Rotem-Galili Y, Dickstein R, Giladi $\mathrm{N}$, Hausdorff JM. Effects of explicit prioritization on dual task walking in patients with Parkinson's disease. Gait Posture. 2012;35(4):641-6. doi: 10.1016/j. gaitpost.2011.12.016.

30. Coelho MS, Patrizzi L, Oliveira APR. Impacto das alterações motoras nas atividades de vida diária na Doença de Parkinson. Revista Neurociencias 2006; 14(10):178-81.

31.Arias P, Cudeiro J. Effect of rhythmic auditory stimulation on gait in Parkinsonian patients with and without freezing of gait. PLoS One. 2010;5(3):e9675. doi: 10.1371/journal.pone.0009675.

32. Nombela C, Hughes LE, Owen AM, Grahn JA. Into the groove: can rhythm influence Parkinson's disease? 
Neurosci Biobehav Rev. 2013 Dec;37(10 Pt 2):2564-70. doi: 10.1016/j.neubiorev.2013.08.003.

33. Benoit CE, Dalla Bella S, Farrugia N, Obrig H, Mainka S, Kotz SA. Musically cued gait-training improves both perceptual and motor timing in Parkinson's disease. Front Hum Neurosci. 2014 Jul 7;8:494. doi: 10.3389/ fnhum.2014.00494.

Recebido em: 13/09/2017

Aceito em: 22/02/2018

Tabela 1. Perfil demográfico dos pacientes com Doença de Parkinson

\begin{tabular}{cccccccccc}
\hline Amostra & Idade & Gr & Estudo & HY & UPDRS & PAS & EEB & MEEM & MoCA \\
\hline 1 & 66 & $\mathrm{~F}$ & $1^{\mathrm{o}}$ grau & 1 & 18 & 43 & 56 & 25 & 24 \\
2 & 64 & $\mathrm{~F}$ & $1^{\mathrm{o}}$ grau & 3 & 31 & 21 & 51 & 27 & 19 \\
3 & 65 & $\mathrm{M}$ & $1^{\mathrm{o}}$ grau & 3 & 30 & 41 & 56 & 27 & 25 \\
4 & 49 & $\mathrm{~F}$ & $1^{\mathrm{o}}$ grau & 3 & 51 & 33 & 50 & 25 & 19 \\
5 & 50 & $\mathrm{~F}$ & Superior & 4 & 43 & 25 & 54 & 30 & 30 \\
\hline Média \pm & & & & & & & & & \\
\hline DP & $58,8 \pm 8,5$ & & & $2,8 \pm 1,10$ & $34,6 \pm 12,74$ & $32,6 \pm 9,63$ & $53,4 \pm 2,79$ & $26,8 \pm 2,05$ & $23,4 \pm 4,62$ \\
\hline
\end{tabular}

HY- Hoehn Yad; UDPDS - Escala Unificada de Avaliação da Doença de Parkinson; PAS - Escala de Atividade de Parkinson; EEB- Escala de Equilíbrio de Berg; MEEM - Mini-Exame do Estado Mental; MoCA - Avaliação Cognitiva Montreal.

Tabela 2. Parâmetros espaço-temporais da marcha nas condições de dupla tarefa sem música e música popular brasileira

\begin{tabular}{|c|c|c|c|}
\hline \multicolumn{4}{|c|}{ Velocidade } \\
\hline & Sem música & Samba & $p$ \\
\hline TUG S & $36,60 \pm 29,45(6-67)$ & $45,20 \pm 23,88(4-62)$ & 0,54 \\
\hline TUG cog & $40,00 \pm 21,08(3-56)$ & $43,60 \pm 9,65(31-56)$ & 0,54 \\
\hline TUG mot & $33,60 \pm 19,20(3-53)$ & $22,80 \pm 17,65(3-42)^{*}$ & 0,85 \\
\hline$p$ & 0,81 & 0,01 & \\
\hline \multicolumn{4}{|c|}{ Comprimento de Passo } \\
\hline & Sem música & Samba & $p$ \\
\hline TUG S & $32,00 \pm 16,61(3-45)$ & $36,80 \pm 3,63(31-41)$ & 0,85 \\
\hline TUG cog & $26,60 \pm 13,08(5-38)$ & $38,00 \pm 15,66(3-42)$ & 0,77 \\
\hline TUG mot & $26,40 \pm 5,22(21-32)$ & $26,60 \pm 5,59(21-33)^{* *}$ & 0,94 \\
\hline$p$ & 0,19 & 0,04 & \\
\hline \multicolumn{4}{|c|}{ Cadência } \\
\hline & Sem música & Samba & $p$ \\
\hline TUG S & $1,56 \pm 0,12(1,40-1,73)$ & $1,42 \pm 0,14(1,23-1,54)$ & 0,54 \\
\hline TUG cog & $1,27 \pm 0,21(1,00-1,67)$ & $1,31 \pm 0,04(1,26-1,38)$ & 0,24 \\
\hline TUG mot & $1,46 \pm 0,15(1,25-1,67)$ & $1,39 \pm 0,13(1,26-1,59)$ & 0,13 \\
\hline$p$ & 0,16 & 0,81 & \\
\hline
\end{tabular}

Resultados expressos pela média \pm desvio padrão (mínimo- máximo). TUG S- Teste Timed Up and Go sem tarefa associada; TUG C- Teste Timed Up and Go associado a tarefa cognitiva; TUG M- Teste Timed Up and Go associado a tarefa motora. *post hoc estatisticamente diferente de TUG S e TUG C; **post hoc estatisticamente diferente de TUG S. 